\title{
Maximum Likelihood Speed and Distance Estimation for OFDM Radar
}

\author{
Martin Braun*, Christian Sturm ${ }^{\ddagger}$ and Friedrich K. Jondral* \\ ${ }^{*}$ Communications Engineering Lab, Karlsruhe Institute of Technology (KIT), Germany \\ ${ }^{\ddagger}$ Institut für Hochfrequenztechnik und Elektronik, Karlsruhe Institute of Technology (KIT), Germany \\ \{martin.braun, friedrich.jondral\}@kit.edu, christian.sturm@ihe.uka.de
}

\begin{abstract}
A distance and speed estimation algorithm for OFDM based radar is analysed statistically. The maximum likelihood estimator is derived and compared to previous results. A connection to spectral analysis is drawn, simplifying the analysis of the estimator in question. Finally, a method to evaluate the performance of the algorithm is presented and conclusions for the structure of the OFDM signals are drawn. It is shown that the estimation algorithm works well above an SNR threshold, but rapidly degrades below.
\end{abstract}

\section{INTRODUCTION}

OFDM radar is a new technique enabling the joint use of radio systems for both communications and radar purposes. Small packets of data are transmitted just as in an 802.11atype OFDM network. The echo of the transmitted signal is simultaneously received and processed to create a radar image of the surroundings.

The idea is not entirely new: [1] and [2] among others discuss aspects and possibilities of combining radar and communication in OFDM systems. [3] proposes a new algorithm, which enables a very efficient calculation of the radar image.

In [4], the question was discussed how to parametrize the OFDM packets such that they are optimal for both applications. One aspect, however, is not covered: which effect does the signal-to-noise ratio at the receiver have concerning the radar system? Since the OFDM signal's parameters depend on this question, the OFDM radar system is analysed in greater depth from the viewpoint of estimation theory, and the connection between the algorithm in [3] and maximum likelihood estimation is drawn.

This paper is organized as follows: In Section II, some assumptions are made and a system model of the OFDM radar is described in detail. Section III derives the maximum likelihood estimator for speed and distance estimation of a single target. An efficient implementation of this estimator is presented in Section IV by comparing the estimation to a known spectral estimation problem. The statistical performance of such an estimator is then evaluated analytically in Section V. Section VI concludes.

\section{System MODEL}

Assume the following setup: when acquiring a radar image, an OFDM signal is sent and the receiver is active at the same time. The distance and the speed of the target cause a propagation delay $\tau$ and a Doppler shift $f_{D}$ of the received signal; target estimation is the process of gathering these two parameters from the received signal.

Perfect isolation between transmit and receive antennas is assumed, and no other signal sources are active during transmission. As a result, the only received signal is the transmitted signal after being reflected by the targets. The receive and transmit front-ends are assumed to be ideal, i.e. they introduce no non-linearities of any kind, and the only distortion is additive white Gaussian noise (AWGN).

Every OFDM frame consists of $N$ sub-carriers and $M$ OFDM symbols. The $l$-th OFDM symbol contains $N$ modulation symbols $c_{k, l} \in \mathcal{A}$, where $\mathcal{A} \subset \mathbb{C}$ is a complex modulation alphabet. For the OFDM modulation, the following parameters are necessary:

- $\Delta f:$ the sub-carrier distance. The frequency of the $k$-th sub-carrier is denoted by $f_{k}=f_{0}+k \Delta f$.

- $T_{G}$ : the length of the guard interval.

- $T_{O}=1 / \Delta f+T_{G}$ : the total duration of one OFDM symbol.

See [4] on how to choose the parametrization. For the scope of this paper, the following assumptions are made:

1) $T_{G}$ is larger than the propagation time of the reflected signal to the furthest target and back.

2) $\Delta f$ is at least one order of magnitude larger than the largest Doppler shift.

3) The signal's centre frequency is several orders of magnitude larger than its total bandwidth, so the Doppler shift is assumed constant over the entire bandwidth.

4) The target speed is small enough to allow for the assumption that its position can be assumed constant during one measurement.

Unlike most publications on OFDM, the frame signal shall be described by an $N \times M$-matrix

$$
\mathbf{F}_{\mathrm{Tx}}=\left(\begin{array}{ccc}
c_{0,0} & \cdots & c_{0, M-1} \\
c_{1,0} & \cdots & c_{1, M-1} \\
\vdots & \ddots & \vdots \\
c_{N-1,0} & \cdots & c_{N-1, M-1}
\end{array}\right) .
$$

In plain language, every row of the matrix corresponds to the data on one sub-carrier, whereas every column corresponds to the data on one OFDM symbol. Two additional assumptions are made towards the transmitted data:

5) The entries of $\mathbf{F}_{\mathrm{Tx}}$ are uncorrelated. 
6) The modulation system is normalized to unit power, i.e. $\left.\left.\mathrm{E}\left\{\mid\left(\mathbf{F}_{\mathrm{Tx}}\right)_{k, l}\right)\right|^{2}\right\}=1$. This is always the case for constant-modulus alphabets such as phase shift keying (PSK).

For the radar imaging process, a time domain signal $s(t)$ is transmitted. Since this is an OFDM system, $s(t)$ is created as follows: Every column of $\mathbf{F}_{\mathrm{Tx}}$ is transformed by an FFT of length greater than $N$ and the result is prepended by a cyclic prefix. The results for the individual columns are then concatenated.

During transmission, the receiver is active. If at least one reflecting target is in range, a time- and frequency-shifted signal is received. This received noisy time domain signal is

$$
r(t)=\sum_{h=0}^{H-1} b_{h} s\left(t-\tau_{h}\right) e^{j 2 \pi f_{D, h} t}+w_{\sigma^{2}}(t) .
$$

$H$ is the number of reflecting targets. $b_{h}=\left|b_{h}\right| e^{j \tilde{\varphi}_{h}}$ is a complex attenuation factor for the $h$-th target. Without loss of generality, assume $\left|b_{0}\right|=1 . w_{\sigma^{2}}(t)$ is complex white Gaussian noise of variance $\sigma^{2}$

The effect of the Doppler shift and the delay on the matrix in (1) can be summarized as follows:

- The Doppler shift causes a row-wise oscillation of the form with the frequency $f_{D, 0}$

- The delay causes a phase shift of $e^{-j 2 \pi\left(f_{0}+k \Delta f\right) \tau_{0}}$.

The backscattered transmit matrix for the first target is thus modified into the received matrix

$$
\left(\mathbf{F}_{\mathrm{Rx}}\right)_{k, l}=\left(\mathbf{F}_{\mathrm{Tx}}\right)_{k, l} \cdot e^{j 2 \pi l T_{O} f_{D, 0}} e^{-j 2 \pi k \tau_{0} \Delta f} e^{j \varphi_{0}}+(\mathbf{W})_{k, l}
$$

$\mathbf{W}$ is the matrix representation of the AWGN; its entries are i.i.d. random values from a circular, complex, zero-mean normal distribution with variance $\sigma^{2}$. All phase shifts which are constant for the entire frame are summarized into the phase term $\varphi_{0}$.

The first component of (3), the modulation data, is known and can therefore be eliminated before estimating delay and frequency shift by dividing $\mathbf{F}_{\mathrm{Rx}}$ element-wise with the transmitted signal

$$
(\mathbf{F})_{k, l}=\frac{\left(\mathbf{F}_{\mathrm{Rx}}\right)_{k, l}}{\left(\mathbf{F}_{\mathrm{Tx}}\right)_{k, l}}=e^{j\left(2 \pi\left(l T_{O} f_{D, 0}-k \tau_{0} \Delta f\right)+\varphi_{0}\right)}+\frac{(\mathbf{W})_{k, l}}{\left(\mathbf{F}_{\mathrm{Tx}}\right)_{k, l}}
$$

This final matrix is passed on to the estimator for the delay $\tau$ and the Doppler shift $f_{D}$ of the targets. Two remarks concerning (4) are in order: first, the noise matrix is transformed into a new noise matrix, where each entry has a new variance of

$$
\sigma_{k, l}^{2}=\operatorname{var}\left\{\frac{(\mathbf{W})_{k, l}}{\left(\mathbf{F}_{\mathrm{Tx}}\right)_{k, l}}\right\}=\frac{\sigma^{2}}{\left|\left(\mathbf{F}_{\mathrm{Tx}}\right)_{k, l}\right|^{2}},
$$

which will be necessary when deriving the likelihood function. Second, (3) indicates that the estimation of $\tau$ and $f_{D}$ is equivalent to estimating frequencies of two orthogonal complex sinusoidal modulations in a matrix - one row-wise modulation caused by the Doppler shift, and one column-wise modulation

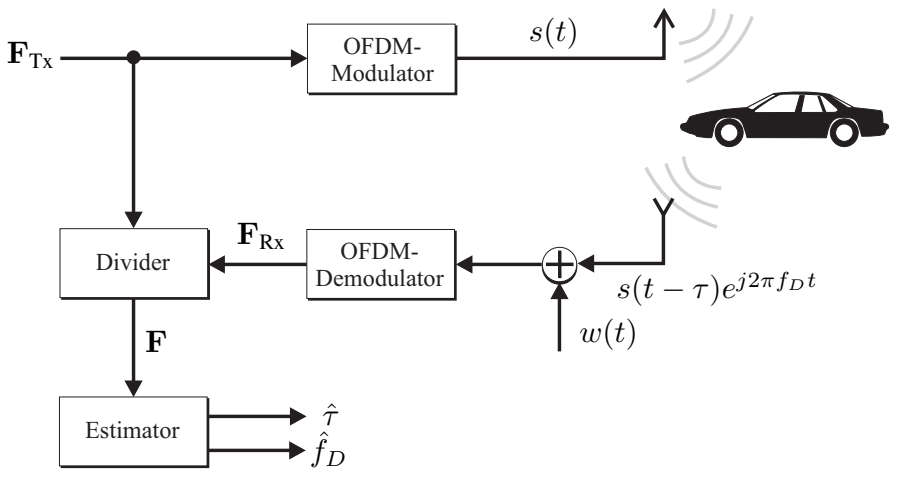

Fig. 1. OFDM Radar System Setup

Below

TABLE I

RADIO SYSTEM PROPERTIES

\begin{tabular}{c|c|c|c|c|c}
\hline$B$ & $f_{C}$ & $G$ & $\mathrm{NF}$ & $T$ & $\sigma_{\mathrm{RCS}}$ \\
\hline $91.5 \mathrm{MHz}$ & $24 \mathrm{GHz}$ & $10 \mathrm{~dB}$ & $20 \mathrm{~dB}$ & $290 \mathrm{~K}$ & $10 \mathrm{~m}^{2}$ \\
\hline
\end{tabular}

caused by the propagation delay. It also follows that the estimation of $\tau$ and $f_{D}$ are two orthogonal problems and can be treated separately.

The entire system setup is shown in Figure 1.

\section{A. Signal-to-noise ratio}

Signal-to-noise ratio (SNR) shall here be defined as the power of the strongest received signal divided by the noise power. Due to the normalization to unit signal power, SNR is defined by the noise power

$$
\mathrm{SNR}=-10 \log _{10} \sigma^{2} .
$$

To estimate the SNR for a given distance, the point-scatter model is used to calculate the received power

$$
P_{\mathrm{Rx}}=\frac{P_{\mathrm{Tx}} G c^{2} \sigma_{\mathrm{RCS}}}{(4 \pi)^{3} f_{C}^{2} r^{4}} .
$$

$P_{\mathrm{Tx}}$ is the transmit power, $G$ is the total antenna gain (on receive and transmit paths), $c$ the speed of light and $\sigma_{\mathrm{RCS}}$ the radar cross section of the target. $f_{C}$ is the signal's centre frequency and $r$ is the distance of the closest target.

Noise power shall be defined by thermal noise plus a noise figure NF. SNR is thus

$$
\mathrm{SNR}=10 \log _{10} \frac{P_{\mathrm{Rx}}}{k_{B} T B \cdot \mathrm{NF}},
$$

where $k_{B}$ is the Boltzmann constant, $B$ is the signal bandwidth, $T$ the receiver temperature and NF the receivers noise figure. For any SNR calculations in this paper, the values from Table I are used, which are based on the values in [4]. The high noise figure is introduced to accommodate for the tough hardware requirements: for a range of several hundred metres, the receiver front-end must work linearly over an extremely high dynamic range, which is a difficult task to accomplish. Radio front-end implementations are not discussed here. 


\section{MAXimum LiKelihood Estimation}

First, an estimator is derived for the case where $H$ is exactly one, and there is no prior information about the target. First of all, a parameter vector is defined as

$$
\boldsymbol{\theta}=\left(\tau, f_{D}, \varphi\right)
$$

This vector also includes the phase offset, which is not required for the radar image. However, since it is unknown, it affects the estimation process, as will be seen later on.

The derivation of a maximum likelihood estimator requires the likelihood function for $\mathbf{F}$ for a given parameter vector. Assumption 5) ensures the noise is uncorrelated, which yields a likelihood function of

$$
f(\mathbf{F} \mid \boldsymbol{\theta})=\prod_{k=0}^{N-1} \prod_{l=0}^{M-1} \frac{1}{\pi \sigma_{k, l}^{2}} e^{-\frac{\left|(\mathbf{F})_{k, l}-e^{j\left(2 \pi\left(l T_{O} f_{D}-k \tau \Delta f\right)+\varphi\right)}\right|^{2}}{\sigma_{k, l}^{2}}} .
$$

The maximization process is simplified when the loglikelihood function is considered instead and the variances are expressed as in (5), yielding

$$
\begin{aligned}
\ell(\mathbf{F} \mid \boldsymbol{\theta}) & =\sum_{k=0}^{N-1} \sum_{l=0}^{M-1}\left(-\log \pi \frac{\sigma^{2}}{\left|\left(\mathbf{F}_{\mathrm{Tx}}\right)_{k, l}\right|^{2}}-\right. \\
& \left.\frac{\left|\left(\mathbf{F}_{\mathrm{Tx}}\right)_{k, l}\right|^{2}}{\sigma^{2}}\left|(\mathbf{F})_{k, l}-e^{j\left(2 \pi\left(l T_{O} f_{D}-k \tau \Delta f\right)+\varphi\right)}\right|^{2}\right) .
\end{aligned}
$$

This function can be simplified further. The first term does not depend on any of the estimation parameters and does thus not affect maximization; it can safely be ignored. The same goes for the constant positive factor $1 / \sigma^{2}$ in the second term. Since every element of $\left|\left(\mathbf{F}_{\mathrm{Tx}}\right)_{k, l}\right|^{2}$ is constant and positive for the entire estimation, it may also be omitted for the maximization. Indeed, by applying assumption 6$), \ell(\mathbf{F} \mid \boldsymbol{\theta})$ will have exactly the same maximum value with or without $\mathbf{F}_{\mathrm{Tx}}$.

The modulus squared is further evaluated using the identity $|a|^{2}=a^{*} a$, where $a^{*}$ denotes the complex conjugate:

$$
\begin{aligned}
& \left|(\mathbf{F})_{k, l}-e^{j\left(2 \pi\left(l T_{O} f_{D}-k \tau \Delta f\right)+\varphi\right)}\right|^{2}= \\
& \left|(\mathbf{F})_{k, l}\right|^{2}+1-2 \operatorname{Re}\left\{(\mathbf{F})_{k, l} e^{-j\left(2 \pi\left(l T_{O} f_{D}-k \tau \Delta f\right)+\varphi\right)}\right\}
\end{aligned}
$$

Again, the constant terms can be ignored for maximization. The simplified log-likelihood function is thus

$$
\tilde{\ell}(\mathbf{F} \mid \boldsymbol{\theta})=\sum_{k=0}^{N-1} \sum_{l=0}^{M-1} \operatorname{Re}\left\{(\mathbf{F})_{k, l} e^{-j\left(2 \pi\left(l T_{O} f_{D}-k \tau \Delta f\right)+\varphi\right)}\right\} .
$$

The maximum likelihood estimate (MLE) for the parameter vector is

$$
\hat{\boldsymbol{\theta}}_{\mathrm{ML}}=\underset{\boldsymbol{\theta}}{\arg \max } \tilde{\ell}(\mathbf{F} \mid \boldsymbol{\theta})
$$

\section{ESTIMATOR IMPLEMENTATION}

The question remains how the MLE can be calculated in accordance with (14). Directly calculating the MLE from (13) would require solving $\nabla_{\boldsymbol{\theta}} \ell\left(\mathbf{F} \mid \hat{\boldsymbol{\theta}}_{\mathrm{ML}}\right)=\mathbf{0}$ which is anything but trivial as $\hat{\boldsymbol{\theta}}_{\mathrm{ML}}$ is only given implicitly in a non-linear set of equations.

A simpler approach can be found by rearranging (13):

$\tilde{\ell}(\mathbf{F} \mid \boldsymbol{\theta})=\operatorname{Re}\left\{e^{j \varphi} \sum_{k=0}^{N-1}\left(\sum_{l=0}^{M-1}(\mathbf{F})_{k, l} e^{-j 2 \pi l T_{O} f_{D}}\right) e^{j 2 \pi k \tau \Delta f}\right\}$

Both the inner and the outer sum share a high resemblance with discrete Fourier transform (DFT). A fast Fourier transform (FFT) based approach can be found when searching for a maximum of $\boldsymbol{\theta}$ on a discrete grid. Quantize $\tau$ and $f_{D}$ as follows:

$$
\begin{aligned}
\tau_{Q, n} & =\frac{n}{N_{\mathrm{FFT}} \Delta f}, n=0, \ldots, N_{\mathrm{FFT}}-1 \\
f_{D, Q, m} & =\frac{m}{M_{\mathrm{FFT}} T_{O}}, m=-\frac{M_{\mathrm{FFT}}}{2}, \ldots, \frac{M_{\mathrm{FFT}}}{2}-1
\end{aligned}
$$

Note that since the Doppler shift can be negative, $m$ is shifted symmetrically around zero.

The goal of the algorithm is to find the discrete values which are closest to the real values $f_{D, 0}$ and $\tau_{0}$. By inserting (16) and (17) into (15), the application of FFTs can be seen immediately:

$$
\ell_{Q}\left(\mathbf{F} \mid \tau_{Q, n}, f_{D, Q, m}, \varphi\right)=\operatorname{Re}\left\{e^{j \varphi} A(n, m)\right\}
$$

where

$$
A(n, m)=\underbrace{\sum_{k=0}^{N_{\mathrm{FFT}}-1} \overbrace{\left(\sum_{l=0}^{M_{\mathrm{FFT}}-1}(\mathbf{F})_{k, l} e^{-j 2 \pi \frac{l m}{M_{\mathrm{FFT}}}}\right)}^{\text {FFT of length } M_{\mathrm{FFT}}} e^{j 2 \pi \frac{k n}{N_{\mathrm{FFT}}}}}_{\text {IFFT of length } N_{\mathrm{FFT}}} .
$$

Choosing a value of $M_{\mathrm{FFT}}>M$, and, respectively, $N_{\mathrm{FFT}}>$ $N$, will increase the accuracy of the estimation at high SNR values, as will be seen in the following section. However, for the scope of this work, $M_{\mathrm{FFT}}$ and $N_{\mathrm{FFT}}$ will be set to $M$ and $N$, respectively.

Finally, the phase shift needs to be taken care of. As hinted before, the target estimation is an identical problem to parameter estimation of complex sinusoids in time-discrete signals. The latter is a well-researched topic and [5] analyses the statistical properties of maximum likelihood estimation of frequency, phase and amplitude of such signals. Applying the argumentation of [5], it can directly be seen that the MLE for $\tau$ and $f_{D}$ (which both affect the frequency of the oscillations) for an unknown phase shift is that which maximizes $|A(n, m)|$. In terms of FFTs, the maximization is thus performed over the function

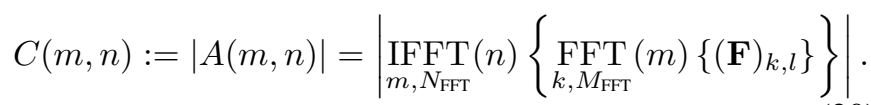


(I)FFT ${ }_{k, L}\{x(k)\}$ denotes a length- $L$ (inverse) FFT of $x(k)$ with respect to $k$. In the equation above, the element-wise matrix product is first subject to an FFT on every column, the result is then subject to an IFFT on every row.

From assumptions 1) and 2), the resulting matrix can be cropped prior to further processing. If $G$ is the fraction of the OFDM symbol used as cyclic prefix, and $D$ is the fraction of the sub-carrier distance the Doppler shift can maximally be, then maximum values for $\hat{n}$ and $\hat{m}$ are defined as

$$
\begin{aligned}
m_{\max } & =\left\lceil D \cdot M_{\mathrm{FFT}}\right\rceil, \\
n_{\max } & =\left\lceil G \cdot N_{\mathrm{FFT}}\right\rceil .
\end{aligned}
$$

The ranges $-m_{\max } \ldots m_{\max }$ and $0 \ldots n_{\max }$ shall be called search ranges. Typical values are $G=1 / 4$ and $D=1 / 10$.

In effect, the result is an extremely simple algorithm for the target estimation, identical to the one proposed in [3]:

1) Find the values $\hat{n}, \hat{m}$ which maximize $C(m, n)$

2) Insert these values into (16) and (17) and solve for $\tau$ and $f_{D}$ to obtain the estimations.

The computational complexity of the algorithm is quite manageable; it consists of

- $N$ FFTs of length $M_{\mathrm{FFT}}$,

- $M$ IFFTs of length $N_{\mathrm{FFT}}$,

- calculating the modulus of $2 m_{\max } \cdot n_{\max }$ complex values,

- finding the largest value from $2 m_{\max } \cdot n_{\max }$ real scalars.

Further optimizations to reduce the algorithm's complexity are possible, but are not discussed here.

\section{Estimator Performance}

Since there are no explicit equations for calculating the estimates from the received values, additional steps have to be taken in order to evaluate the performance of the estimator in dependence of SNR. The following derivation is similar to that in [5], but with less approximations.

To determine the estimator performance, the discrete probability density functions (PDF) for $\hat{m}$ and $\hat{n}$ are derived. First, introduce two variables $m_{0}=f_{D, 0} T_{O} M_{\mathrm{FFT}}$ and $n_{0}=$ $\tau_{0} \Delta f N_{\mathrm{FFT}}$. Inserting these and (4) into $C(m, n)$ yields

$$
\begin{gathered}
C(m, n)=\mid \overbrace{\sum_{k=0}^{N_{\mathrm{FFT}}-1}\left(\sum_{l=0}^{M_{\mathrm{FFT}}-1} e^{-j 2 \pi l \frac{m-m_{0}}{M_{\mathrm{FFT}}}}\right) e^{j 2 \pi k \frac{n-n_{0}}{N_{\mathrm{FFT}}}}}^{\text {Signal term }}+ \\
\underbrace{\sum_{k=0}^{N_{\mathrm{FFT}}-1}\left(\sum_{l=0}^{M_{\mathrm{FFT}}-1}\left(\mathbf{F}_{\mathrm{Tx}}\right)_{k, l}^{-1}(\mathbf{W})_{k, l} e^{-j 2 \pi \frac{l m}{M_{\mathrm{FFT}}}}\right) e^{j 2 \pi \frac{k n}{N_{\mathrm{FFT}}}}}_{\text {Noise term }} \mid .
\end{gathered}
$$

The phase shift in the received signal $\varphi_{0}$ can be ignored due to the rotational invariance of the noise.

From Section II it follows that $m$ and $n$ can be estimated separately. By summing up over all possible values of $n$, the dependence of $n$ is removed from (22)

$$
\begin{aligned}
C(m) & =\frac{1}{M N}\left|\sum_{n} A(m, n)\right| \\
& =\left|a(m)+w_{\sigma^{2} /(N M)}(m)\right|,
\end{aligned}
$$

where $a(m)$ is the amplitude of the $m$-th FFT bin in the noisefree case,

$$
a(m)=\left|\frac{\sin \left(\pi M \frac{m-m_{0}}{M_{\mathrm{FFT}}}\right)}{M \sin \left(\pi \frac{m-m_{0}}{M_{\mathrm{FFT}}}\right)}\right| .
$$

$C(m)$ is normalized by $M N$ so that $a(m)$ has unit value at its peak. This reduces the noise power to $\sigma^{2} /(M N)$, effectively increasing SNR in the FFT bin closest to $m_{0}$. This is to be expected, since the double FFT algorithm correlates over the entire bandwidth and signal duration.

Every $C(m)$ is a random variable with a Rician distribution of the form

$$
f_{C(m)}(x)=\frac{2 M N x}{\sigma^{2}} e^{-\frac{M N\left(x^{2}+a^{2}(m)\right)}{\sigma^{2}}} I_{0}\left(\frac{2 M N x a(m)}{\sigma^{2}}\right),
$$

where $x \geq 0$ and $I_{0}(x)$ denotes the modified Bessel function of order zero.

The estimator result is also a random variable and shall be denoted by $\hat{\mathbf{m}}$. Its PDF is given by $f_{\hat{\mathbf{m}}}\left(m \mid m_{0}\right)$ and depends on $m_{0}$. From all $m$ within the search range, the estimator decides for the particular value $\hat{m}$ whenever $C(\hat{m})$ is larger than any other $C(m)$. The probability for this is

$$
\begin{aligned}
f_{\hat{\mathbf{m}}}\left(\hat{m} \mid m_{0}\right) & =P\{C(\hat{m})>C(m) \mid \forall m \neq \hat{m}\} \\
& =\int_{x} P\{C(m)<x \mid m \neq \hat{m}\} f_{C(\hat{m})}(x) d x \\
& =\int_{0}^{\infty} f_{C(m)}(x)\left[\prod_{m \neq \hat{m}} \int_{0}^{x} f_{C(\hat{m})}(y) d y\right] d x \\
& =\int_{0}^{\infty}\left[\prod_{m \neq \hat{m}} F_{C(m)}(x)\right] f_{C(\hat{m})}(x) d x
\end{aligned}
$$

where $F_{C(m)}(x)$ is the cumulative density of the value of $C(m)$,

$$
F_{C(m)}(x)=1-Q_{1}\left(\frac{a(m) \sqrt{2 M N}}{\sigma}, \frac{x \sqrt{2 M N}}{\sigma}\right)
$$

and $Q_{1}(\alpha, \beta)$ is the Marcum Q-function.

The complete PDF for $\hat{\mathbf{m}}$ is calculated for a given $m_{0}$ and SNR by solving the integral for every possible value of $\hat{m}$ in the search range ${ }^{1}$. PDFs for $n$ can be calculated the same way if $m, m_{0}$ and $M$ are swapped with $n, n_{0}$ and $N$, respectively.

${ }^{1}$ (26) can be solved numerically, but requires special attention due to the combination of Bessel and exponential functions, which can have extremely large or small values and can thus become numerically unstable. Numerically demanding calculations were done with the help of the arbitrary precision tools for the Python programming language. The methods used to calculate $Q_{1}(\alpha, \beta)$ are those explained in [6]. 


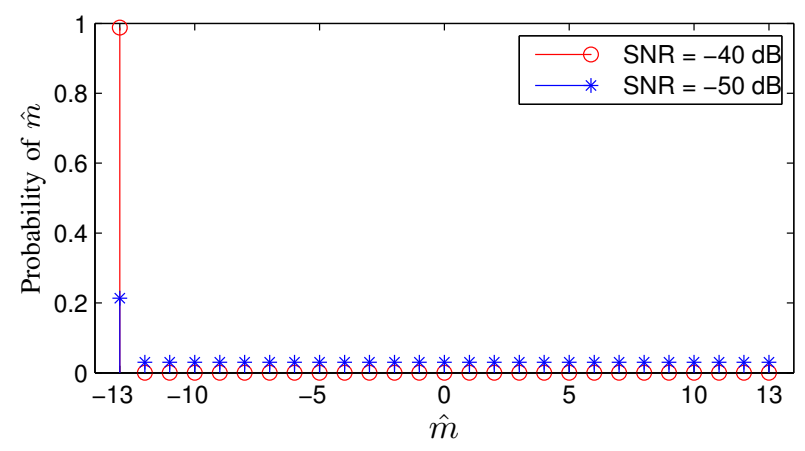

Fig. 2. Examples for discrete PDFs for $m$ and different SNR values

In [5], two important aspects of this estimator are mentioned that affect the performance and thus shall be analysed here. First, the estimator is biased. This follows directly from the generally asymmetric and limited form of $f_{\hat{\mathbf{m}}}\left(m \mid m_{0}\right)$. As an example, assume $m_{0}=-m_{\max }$. The highest probability for the decision value is for $\hat{m}=m_{0}$. However, all other non-zero entries of the PDF shift the expected value towards the middle of the search range, thus introducing a bias. Figure 2 depicts possible PDFs for two values of SNR. As the SNR drops, the probability for any other result than the correct value of $\hat{m}$ rises. While the mode of the PDFs stays at the correct value $m_{0}$, the mean shifts towards the right.

The other aspect is the threshold effect. Above a specific SNR value, subsequently called the SNR threshold, the estimator works very well. When SNR drops below the threshold, the estimator performance will degrade very rapidly. This is the case as well for the OFDM radar estimator. It is important to design the radar system in such a fashion that the estimator does not operate below the threshold.

Bias and standard deviation of speed and distance estimates are used as quality criterion for the estimator. Both can be calculated from the PDFs for $\hat{\mathbf{m}}$ and $\hat{\mathbf{n}}$ by applying (16), (17) and the relations $v=0.5 c f_{D} / f_{c}$ and $r=0.5 \tau c$, resulting in

$$
\begin{aligned}
\operatorname{bias}\{r\} & =\frac{c}{2 N_{\mathrm{FFT}} \Delta f}\left(\mathrm{E}\{\hat{\mathbf{n}}\}-n_{0}\right) \\
\mathrm{D}\{r\} & =\frac{c}{2 N_{\mathrm{FFT}} \Delta f} \sqrt{\mathrm{E}\left\{(\hat{\mathbf{n}}-\mathrm{E}\{\hat{\mathbf{n}}\})^{2}\right\}} \\
\operatorname{bias}\{v\} & =\frac{c}{2 M_{\mathrm{FFT}} T_{O} f_{c}}\left(\mathrm{E}\{\hat{\mathbf{m}}\}-m_{0}\right) \\
\mathrm{D}\{v\} & =\frac{c}{2 M_{\mathrm{FFT}} T_{O} f_{c}} \sqrt{\mathrm{E}\left\{(\hat{\mathbf{m}}-\mathrm{E}\{\hat{\mathbf{m}}\})^{2}\right\}} .
\end{aligned}
$$

As an example, a system with the following parameters shall be analysed: $M=128, N=1200, \Delta f=76.25 \mathrm{kHz}$ and $T_{G}=1 /(4 \Delta f)$. The radio system is parametrized as in Table I. $D$ and $G$ are fixed to $1 / 10$ and $1 / 4$, respectively, resulting in $m_{\max }=7$ and $n_{\max }=300$. The following calculations are performed: at a fixed value of $m_{0}=-7, n_{0}$ is increased in integer steps from 1 to $n_{\max }$. The choice of $m_{0}$ is such that the bias and standard deviation will be maximal.

At every value of $n_{0}$, corresponding distance and, using the values from Table I and (8), SNR are calculated. Having

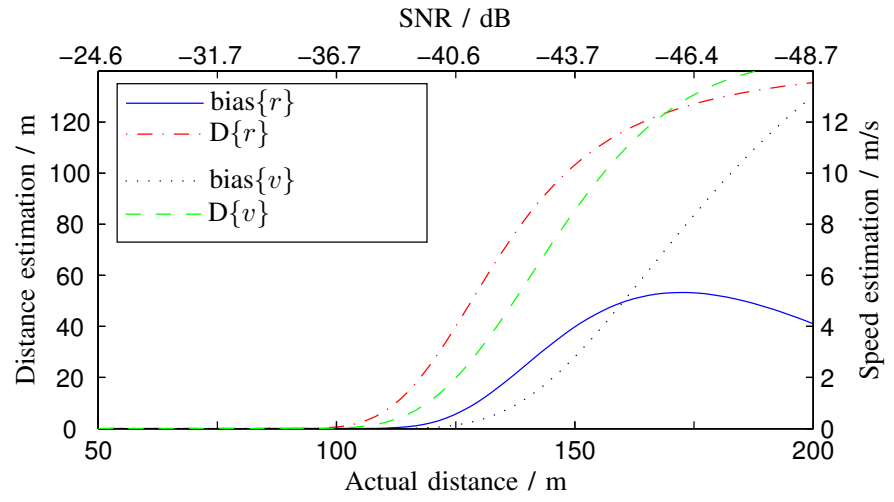

Fig. 3. Performance of target speed and distance estimation against SNR and actual distance

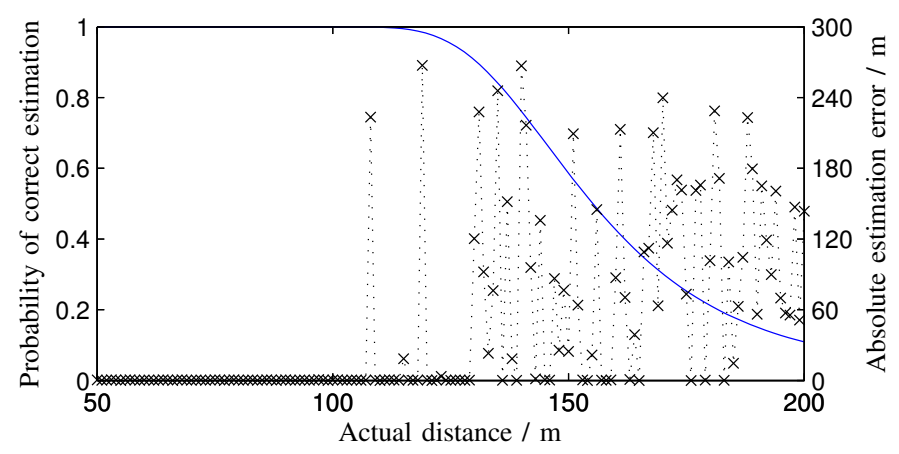

Fig. 4. Probability of correct value estimation (solid line) and simulated estimation error (dotted line)

calculated the latter, PDFs for $\hat{\mathbf{m}}$ and $\hat{\mathbf{n}}$ are derived using (26). Finally, bias and standard deviation are calculated. Figure 3 shows the resulting bias and standard deviation for the given example. Both speed and distance estimations are shown in the same plot. The figure is to be read like this: above approx. $-36.7 \mathrm{~dB}$ SNR, the estimation is very good. Assuming the radio system properties are correct, this corresponds to a distance of about $100 \mathrm{~m}$. When going beyond, the estimates quickly become very unreliable, both for speed and distance. The descent of the distance bias is only due to fact that $n_{0}$ itself approaches $n_{\max } / 2$, where the bias is always zero. It must be clarified that the standard deviation is not a mean error for the estimator, but rather related to the probability of not estimating the correct value. Whenever the estimator makes a wrong choice, the estimated value can be anywhere within the search range. Figure 4 demonstrates this effect for the distance estimation. The solid line shows the probability for the correct bin, the dotted line is the result from a simulated measurement. For high SNR, the estimator error is always near-zero. Below the SNR threshold, the error does not gradually become larger, but rather occurs more often. The error magnitude itself can be of any value.

Above the SNR threshold, another effect becomes more important: $m_{0}$ and $n_{0}$ can be of non-integer value, but the 
estimator always estimates integer values. From (16) and (17), it can be seen that this error can be reduced by increasing $M_{\mathrm{FFT}}$ and $N_{\text {FFT }}$.

\section{A. Consequences for signal design}

The results presented here show a hitherto unmentioned effect of the signal structure, in particular the choice of $M$ and $N$. [4] explains how these parameters affect the radar resolution. The previous section shows how they affect the reliability of the estimation.

To illustrate, see what happens if $N$ is doubled. From (16), it is clear that the distance resolution is improved. It also decreases the noise power in (23) by $3 \mathrm{~dB}$, thereby increasing the reliability of the speed measurement.

A new benchmark for the radar system is thus its SNR performance. For a given radio system setup, a maximum reliable range can be calculated. If targets at greater range should still be reliably estimated, $M$ or $N$ must be increased until the SNR threshold is low enough.

A different part of the signal is the transmitted data itself. Its effects are best studied by showing how it affects (22). In the noise term, the expression $\left(\mathbf{F}_{\mathrm{Tx}}\right)_{k, l}^{-1}(\mathbf{W})_{k, l}$ is given closer inspection. Under the safe assumption that $\mathbf{F}_{\mathrm{Tx}}$ and $\mathbf{W}$ are uncorrelated, the total noise power inside the noise term is equal to $\sigma^{2}$ as a result of assumption 6). In other words, the data modifies the noise into a new noise with exactly the same statistics.

The importance of assumption 5) must be emphasized. If the data are in fact correlated, then the new noise does have different statistics, and the derivation in the previous sections is not valid.

\section{CONCLUSION AND Future WORK}

In this paper, it was shown that the OFDM radar method proposed in [3] is in fact an efficient implementation of the MLE. The statistical performance of such an estimator was analysed, yielding very good estimates above a certain threshold SNR, after which the estimation quality rapidly decays. A method to plot the variance for given SNR rates and thus distances is given in the same Section.
Future research will explore alternative estimators, in particular those which can operate on an arbitrary set of sub-carriers. Also, the question must be answered how the parametrization is optimized when multiple targets are to be expected. Live measurements will complement the results, preliminary measurements already confirm this kind of radar setup works [7].

\section{ACKNOWLEDGEMENTS}

The authors gratefully acknowledge that their work is supported within the priority program No. 1163 (TakeOFDM) by the German Research Foundation (DFG).

\section{REFERENCES}

[1] B. Donnet and I. Longstaff, "Combining MIMO Radar with OFDM Communications," Radar Conference, 2006. EuRAD 2006. 3rd European, pp. 37-40, Sept. 2006.

[2] G. E. A. Franken, H. Nikookar, and P. van Genderen, "Doppler tolerance of OFDM coded Radar Signals," Proc. 3rd European Radar Conference, pp. 108-111, Sep 2006.

[3] C. Sturm, T. Zwick, and W. Wiesbeck, "An OFDM System Concept for Joint Radar and Communications Operations," Vehicular Technology Conference, 2009. 69th IEEE, April 2009.

[4] M. Braun, C. Sturm, A. Niethammer, and F. K. Jondral, "Parametrization of Joint OFDM-based Radar and Communication Systems for Vehicular Applications," 20th IEEE Symposium on Personal Indoor and Mobile Radio Communications, 2009.

[5] D. Rife and R. Boorstyn, "Single tone parameter estimation from discretetime observations," Information Theory, IEEE Transactions on, vol. 20, no. 5, pp. 591-598, Sep 1974.

[6] D. Shnidman, "The calculation of the probability of detection and the generalized Marcum Q-function," Information Theory, IEEE Transactions on, vol. 35, no. 2, pp. 389-400, Mar 1989.

[7] C. Sturm, M. Braun, T. Zwick, and W. Wiesbeck, "Performance Verification of Symbol-Based OFDM Radar Processing," Radar Conference, IEEE International, 2010 